\title{
Spatial segmentation and feature selection for desi imaging mass spectrometry data with spatially-aware sparse clustering
}

\author{
Kyle D Bemis ${ }^{1 *}$, Livia Eberlin², Christina Ferreira ${ }^{2}$, R Graham Cooks ${ }^{2}$, Olga Vitek ${ }^{1,3}$ \\ From Eighth International Society for Computational Biology (ISCB) Student Council Symposium 2012 \\ Long Beach, CA, USA. 13-14 July 2012
}

\section{Background}

Recent experimental advances in matrix-assisted laser desorption/ionization (MALDI) and desorption electrospray ionization (DESI) have demonstrated the usefulness of these technologies in the molecular imaging of biological samples. However, development of computational methods for the statistical interpretation and analysis of the chemical differences present in the distinct regions of these samples is still a major challenge. In this poster, we propose statistically-minded methods and computational tools for analyzing DESI imaging experiments. Specifically, we present techniques for signal processing and unsupervised multivariate image segmentation, which are also applicable to other imaging mass spectrometry (IMS) methods such as MALDI.

\section{Method}

Signal processing of DESI spectra typically involves binning to reduce dimensionality, but this inefficient for downstream analysis as it retains empty regions of the mass spectrum. In our proposed processing step, we apply a novel peak picking algorithm based on windowed smoothing splines that allows adaptive resolution based on spectral profile. With this approach, peaks are aligned using a recursive dynamic programming algorithm which accounts for the heterogenous nature of IMS data by making pairwise alignments between pixels based on their proximity. Peaks are then normalized using total ion count.

In order to segment the sample into sub-regions of homogenous chemical composition in MALDI images,
Alexandrov \& Kobarg [1] proposed two efficient spatiallyaware clustering techniques. We demonstrate that these approaches are also useful for DESI. Moreover, we extend one of these clustering methods using statistical regularization techniques that enable simultaneous feature selection of structurally-important peaks and facilitate interpretation.

\section{Conclusions}

We evaluate the performance of the proposed methods in two applications. First, in a non-biological application of DESI-imaging, we recreate a painting from the clustering of its DESI mass spectra (Figure 1). Since the visual content of the painting is known, it can be used as a gold standard to evaluate the performance of these methods. In the second application, we present the spatial segmentation of a fetal pig section, and evaluate the performance of our methods by the quality of the mapping between the spatial segmentation and the morphological and functional structures (Figure 2). We show that statistical regularization improves accuracy and interpretation of the spatial segmentation over existing approaches.

\footnotetext{
Author details

${ }^{1}$ Department of Statistics, Purdue University, West Lafayette, IN, USA.

${ }^{2}$ Department of Chemistry, Purdue University, West Lafayette, IN, USA.

${ }^{3}$ Department of Computer Science, Purdue University, West Lafayette, IN, USA.
}

Published: 14 December 2012

\section{Reference}

1. Alexandrov $\mathrm{T}$, Kobarg $\mathrm{JH}$ : Efficient spatial segmentation of large imaging mass spectrometry datasets with spatially aware clustering. Bioinformatics 2011, 27(13):i230-238.

* Correspondence: kbemis@purdue.edu

'Department of Statistics, Purdue University, West Lafayette, IN, USA

Full list of author information is available at the end of the article 


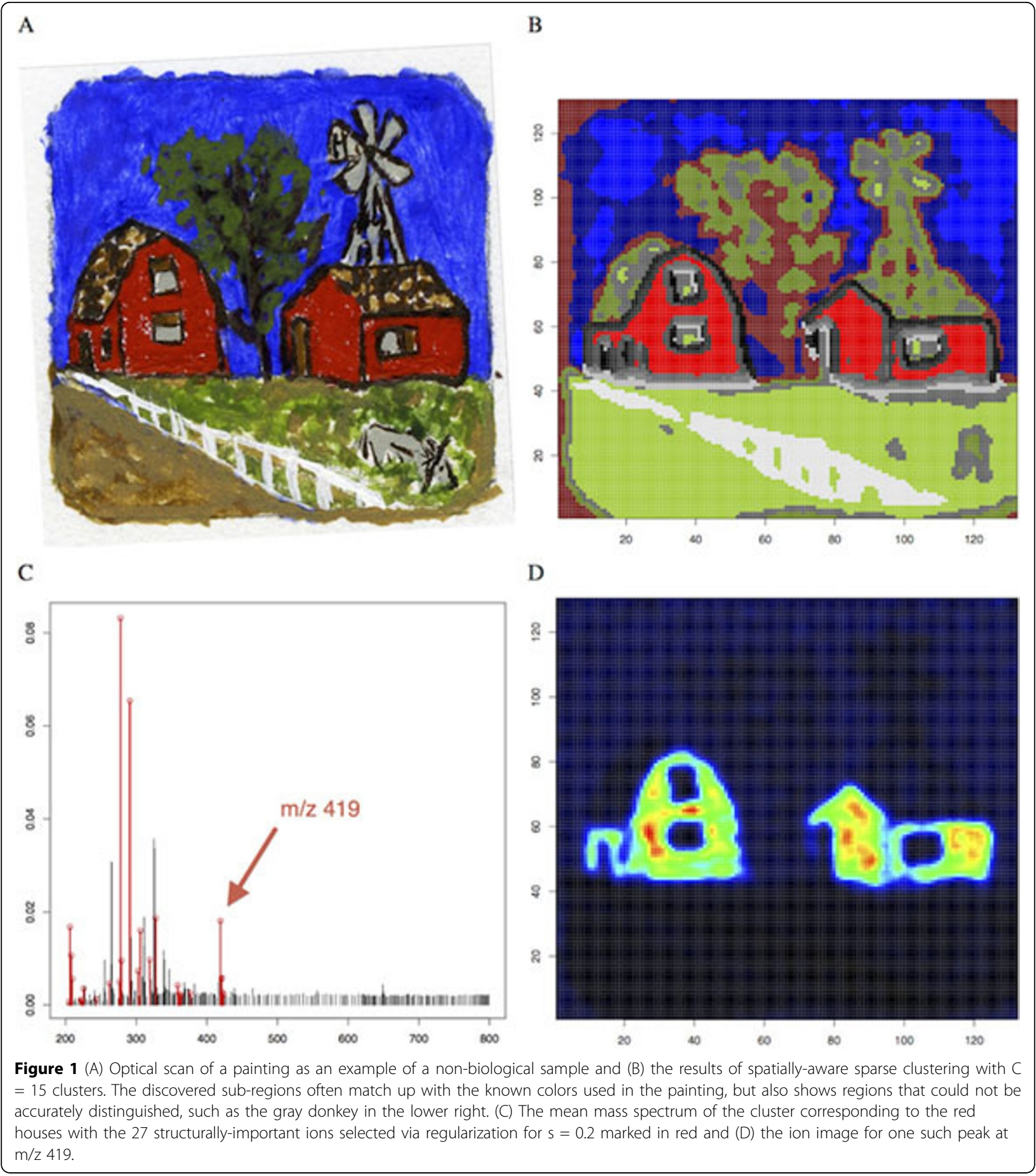




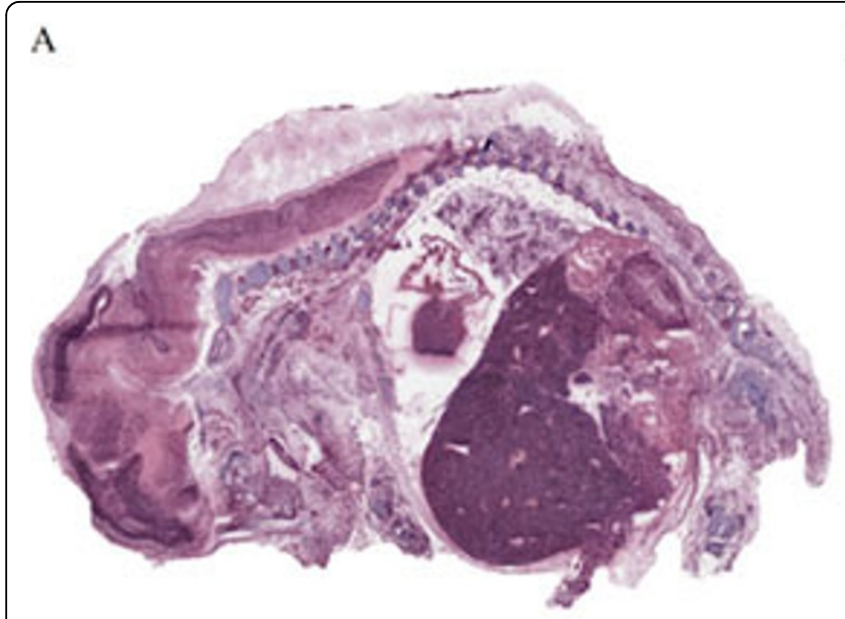

B

$\mathrm{C}$

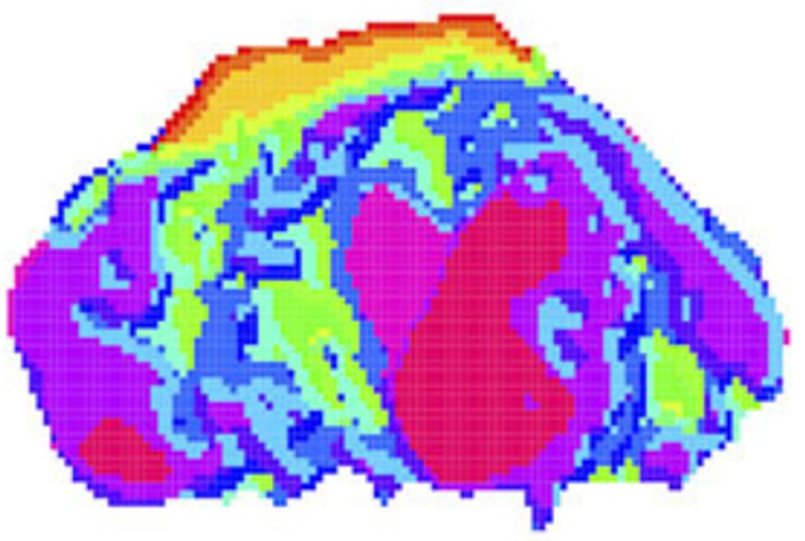

D
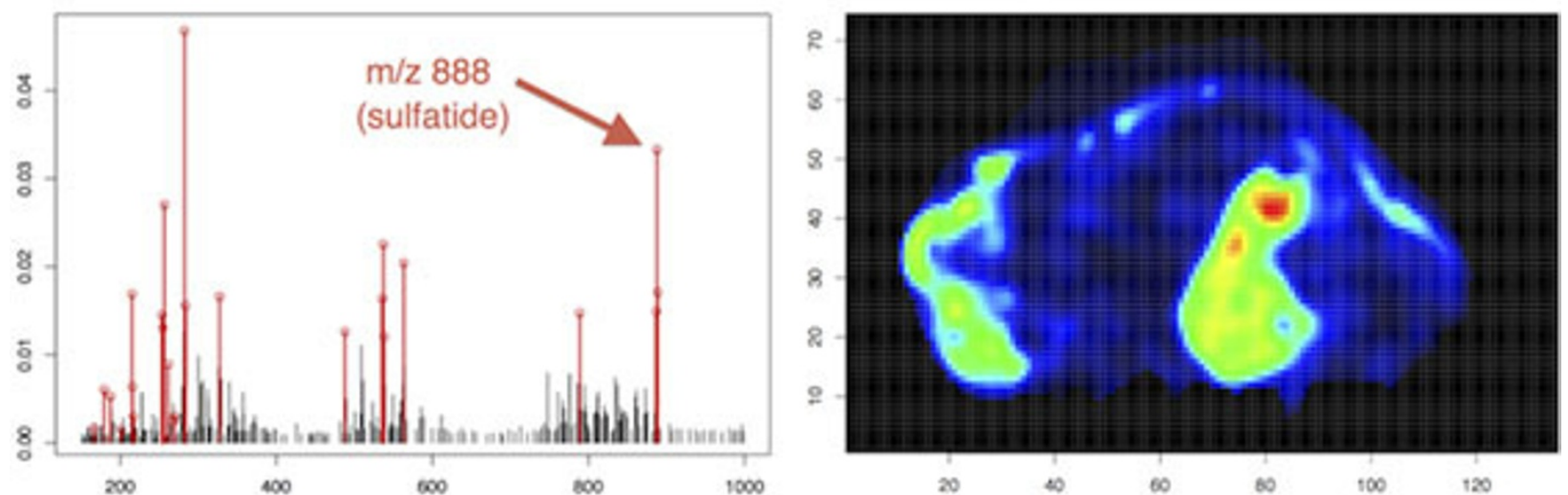

Figure 2 (A) The fetal pig section and (B) the results of spatially-aware sparse clustering with $C=15$ clusters. Note that the clusters are regions of homogenous chemical composition and often match up with visually distinguishable anatomical features, while also revealing possible hidden chemical structures in regions that are visually ambiguous. (C) The mean mass spectrum of the cluster corresponding to the liver and part of the brain with the 25 structurally-important ions selected via regularization for $s=0.6$ marked in red and (D) the ion image for one such peak at $\mathrm{m} / \mathrm{z} 888$.

doi:10.1186/1471-2105-13-S18-A8

Cite this article as: Bemis et al: Spatial segmentation and feature selection for desi imaging mass spectrometry data with spatially-aware sparse clustering. BMC Bioinformatics 2012 13(Suppl 18):A8.

\section{Submit your next manuscript to BioMed Central} and take full advantage of:

- Convenient online submission

- Thorough peer review

- No space constraints or color figure charges

- Immediate publication on acceptance

- Inclusion in PubMed, CAS, Scopus and Google Scholar

- Research which is freely available for redistribution 